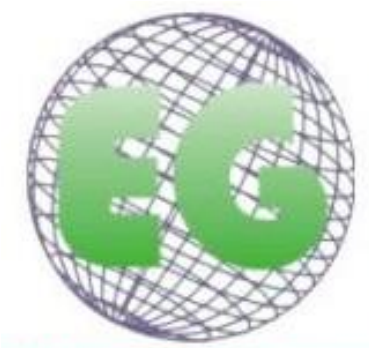

ISSN 1695-6141 $N^{\circ} 51$

\title{
Valores, espiritualidad y consumo de alcohol en estudiantes de preparatoria
}

Values, spirituality and alcohol consumption in high school students

Carmen Leticia Cervantes Peña ${ }^{1}$

Linda Azucena Rodríguez Puente ${ }^{2}$

María Magdalena Alonso Castillo ${ }^{1}$

Nora Nelly Oliva Rodríguez ${ }^{1}$

Alicia Álvarez Martínez ${ }^{3}$

Alejandra Leija Mendoza ${ }^{2}$

\footnotetext{
${ }^{1}$ Facultad de Enfermería, Universidad Autónoma de Nuevo León, Monterrey, México.

${ }^{2}$ Facultad de Enfermería "Dr. Santiago Valdés Galindo" Universidad Autónoma de Coahuila, Saltillo, México.

3 Facultad de Enfermería y Obstetricia, Universidad de Guanajuato, Campus Celaya-Salvatierra, México.
}

E-mail: leti carmen@hotmail.com

http://dx.doi.org/10.6018/eglobal.17.3.284761

Recibido: 09/06/2017

Aceptado: 10/09/2017

\section{RESUMEN:}

Introducción: Los valores y la espiritualidad se han identificado como factores protectores que disminuyen el desarrollo de comportamientos riesgosos en los adolescentes como el consumo de alcohol.

Objetivo: Identificar los valores y la espiritualidad en adolescentes de preparatoria y determinar la relación que existe entre los valores y la espiritualidad con el consumo de alcohol en adolescentes de preparatoria.

Método: Se utilizó un diseño descriptivo correlacional. La población fue de 4728 estudiantes de una preparatoria del área metropolitana de Monterrey, Nuevo León. Se realizó un muestreo probabilístico estratificado unietápico, la muestra estuvo conformada por 317 sujetos inscritos. Las variables se evaluaron mediante el Cuestionario de Valores de Schwartz (VAL), el Cuestionario de Espiritualidad (CE) y el AUDIT.

Resultados: Se encontró que el índice general de valores mostró una media de $70.4(D E=11.1, M d n=$ 70.54), el índice de espiritualidad mostró una media de 66.3 ( $D E=13.4, M d n=67.82)$. Se encontró correlación positiva y significativa entre las creencias espirituales y el índice de consumo dependiente de alcohol $\left(r_{s}=.181, p=.016\right)$, correlación negativa significativa entre las creencias espirituales y la cantidad de bebidas alcohólicas consumidas por ocasión $\left(r_{s}=-.117, p=.038\right)$ y correlación positiva y significativa entre las necesidades espirituales y el índice de consumo dañino $\left(r_{s}=.180, p=.017\right)$.

Conclusiones: Los adolescentes que tienen mayores creencias espirituales y necesidades espirituales presentan mayor consumo de alcohol dependiente y dañino. 
Los adolescentes que presentan mayores creencias espirituales consumen menor cantidad de bebidas alcohólicas por ocasión.

Palabras clave: Valores; Espiritualidad; Consumo de Alcohol; Adolescentes

\section{ABSTRACT:}

Introduction: Values and spirituality have been identified as protective factors that decrease the development of risky behaviors in adolescents such as alcohol consumption.

Aim: To identify values and spirituality in high school adolescents and determine the relationship between values and spirituality with alcohol consumption in high school adolescents.

Method: A descriptive correlational design was used. The population was of 4728 students from a high school in the central area of Monterrey, Nuevo León. It was used a a single-stage stratified probabilistic sampling, the sample consisted of 317 enrolled subjects. The variables were evaluated using the Schwartz Values Questionnaire (VAL), the Spirituality Questionnaire (CE) and the AUDIT.

Results: It was found that the general index of values showed an average of $70.4(S D=11.1, M d n=$ 70.54), the spirituality index showed an average of 66.3 ( $S D=13.4, M d n=67.82$ ). A positive and significant correlation was found between spiritual beliefs and the index of alcohol dependent consumption $\left(r_{s}=.181, p=.016\right)$, a significant negative correlation between spiritual beliefs and the number of alcoholic beverages consumed per occasion $\left(r_{s}=.117, \mathrm{p}=.038\right)$ and positive and significant correlation between spiritual needs and the index of harmful consumption $\left(r_{s}=.180, \mathrm{p}=.017\right)$.

Conclusions: Adolescents who have greater spiritual beliefs and spiritual needs are more dependent on harmful alcohol consumption.

Key words: Values; Spirituality; Alcohol Consumption; Adolescents.

\section{INTRODUCCIÓN}

El consumo de alcohol es un problema de salud pública, contribuye de manera significativa en la carga mundial de morbilidad siendo factor causal de más de 200 enfermedades ${ }^{(1)}$ y representando una proporción considerable de situaciones de riesgo atribuible a la ingesta excesiva de alcohol como accidentes, violencia física, violaciones, conductas sexuales de riesgo, homicidios y suicidios, en el año 2014, se reportaron 3.3 millones de muertes en el mundo por causas relacionadas con el consumo de alcohol, incluyendo a 320,000 jóvenes de entre 15 a 29 años de edad, lo que representa el $5.9 \%$ de la mortalidad a nivel mundial ${ }^{(2)}$.

Según la Encuesta Nacional de Consumo de Drogas, Alcohol y Tabaco (ENCODAT, 2016), en la población de 12 a 17 años el consumo excesivo de alcohol se duplicó, de $4.3 \%$ en 2011 a un $8.3 \%$ en 2016 , observándose un incremento significativo en las mujeres de $2.2 \%$ a 7.7\%. Además, en el año 2016 el 1\% de la población en este grupo de edad presentó consumo consuetudinario de alcohol mientras que en el 2016 se reportó el $4.1 \%$, de los cuales el $4.4 \%$ corresponde a hombres y el $3.9 \%$ a las mujeres, en el consumo diario también hubo un aumento importante de $0.2 \%$ a $2.6 \%$ del año 2011 al 2016 respectivamente ${ }^{(3)}$.

Los adolescentes se encuentran en un proceso de maduración física, psicológica, sexual y social que les permite culminar en el desarrollo de una identidad, a través del conocimiento de sí mismos, sus preferencias, actitudes, limitaciones y motivaciones, además en esta etapa es donde se busca mayor libertad y autonomía, que los convierte en personas vulnerables al consumo de sustancias como el alcohol ${ }^{(4)}$, es por esto que necesita de un conjunto de valores, prácticas y creencias espirituales que guíen su conducta y su comportamiento y así responder a las diferentes situaciones de la vida cotidiana, tomando en cuenta su conjunto se valores y creencias espirituales $^{(5,6)}$. 
Se tiene evidencia de que los valores y la espiritualidad propios de los adolescentes destacan entre los factores protectores al consumo de alcohol de mayor importancia ${ }^{(6-9)}$. Estudios reconocen que estos conceptos juegan un rol importante en esta etapa de transición y disminuyen el desarrollo de comportamientos riesgosos como el consumo de alcohol ${ }^{(10-12)}$.

Se considera que existe un vacío de conocimiento en relación a los valores, la espiritualidad y el consumo de alcohol, ya que hasta el momento no se localizaron estudios donde se relacionen estas tres variables. El profesional de enfermería tiene un rol sumamente importante en la prevención del consumo de drogas como el alcohol. Este estudio aportará conocimiento científico que permitirá al profesional de enfermería desarrollar estrategias de prevención y reducción del consumo de alcohol mediante el fortalecimiento de los valores y la espiritualidad en los adolescentes.

\section{Objetivos específicos}

1. Identificar los valores y la espiritualidad en estudiantes de preparatoria.

2. Determinar la relación que existe entre los valores y la espiritualidad con el consumo de alcohol en adolescentes de preparatoria.

\section{MATERIAL Y MÉTODOS}

\section{Diseño de estudio}

El diseño fue descriptivo-correlacional. La población estuvo conformada por estudiantes de una preparatoria del área metropolitana de Monterrey, Nuevo León, México. Se realizó un muestreo probabilístico estratificado unietápico de una población de 4728 estudiantes.

\section{Participantes}

La muestra estuvo conformada por 317 sujetos inscritos, pertenecientes al $2^{\circ}$ y $4^{\circ}$ semestre. La media de edad de los participantes fue de 15.9 años $(D E=.801)$, predominó el sexo femenino con un $53.9 \%$, el $93.1 \%$ eran solteros, el $62.1 \%$ cursa el segundo semestre de preparatoria, el $85.5 \%$ solo estudia y el $69.4 \%$ manifestó ser de religión católica.

\section{Mediciones}

Datos sociodemográficos. Cédula de Datos Personales e Historial de Consumo de Alcohol (CDPHCA) que consta de 12 preguntas donde se indaga acerca de la edad, sexo, estado civil, nivel educativo, ocupación, religión, considera los tipos de prevalencia de consumo de alcohol, la edad de inicio de consumo y el número de bebidas en un día típico.

Valores. El Cuestionario de Valores de Schwartz (VAL) ${ }^{(13)}$, mide los valores del adolescente, consta de 56 ítems, que se dividen en dos sub escalas: valores terminales e instrumentales. Este instrumento ha sido utilizado en adolescentes mexicanos donde ha reportado Alphas de Cronbach que oscilan entre .90 y .93 .

Espiritualidad. El Cuestionario de Espiritualidad de Parsian y Dunning (ESP) ${ }^{(14)}$, el cual se enfoca en el concepto de identidad interior, sentido de la vida y conexión de las personas jóvenes. Está conformado por 29 ítems divididos en cuatro subescalas: 
autoconciencia, creencias espirituales en la vida, prácticas espirituales y necesidades espirituales. Éste instrumento reportó un Alpha de Cronbach de 0.83.

Se obtuvieron índices de cada subescala y de la escala en general de los dos instrumentos, donde a mayor puntaje mayor es el índice de valores y espiritualidad.

\section{RESULTADOS}

El índice general de valores mostró una media de $70.4(D E=11.1, M d n=70.54)$, el índice de valores terminales tuvo una media de 67.9 ( $D E=11.4, M d n=66.67)$, mientras que el índice de valores instrumentales presentó una media de 74.2 ( $D E=12.6, M d n=$ 75), los valores terminales seleccionados por los participantes fueron dar significado a mi vida y seguridad familiar, mientras que los valores instrumentales elegidos fueron eligiendo mis propias metas y ser exitoso. El índice de espiritualidad mostró una media de $66.3(D E=13.4, M d n=67.82)$, el índice de la subescala autoconciencia tuvo una media de 73.7 ( $D E=15.7, M d n=76.67)$, el índice de creencias espirituales mostró una media de $56.5(D E=25.9, M d n=58.3)$, el índice de prácticas espirituales tuvo una media de $49.5(D E=.20 .8, M d n=50.0)$ y el índice de necesidades espirituales presentó una media de $75.5(D E=14.4, M d n=77.7)$.

La edad de inicio de consumo de alcohol fue de 12.9 años $(D E=.057, M d n=13)$ y la cantidad de bebidas consumidas en un día típico fue en promedio de $2.6(D E=4.3)$. En las prevalencias de consumo de alcohol se encontró que el $71 \%$ de los participantes han consumido alcohol alguna vez en la vida, el $54.9 \%$ ha consumido alcohol en el último año, el $3.8 \%$ ha consumido alcohol en el último mes y el $16.7 \%$ de los participantes han consumido alcohol en los últimos 7 días.

Se encontró que existe correlación positiva significativa entre el índice de frecuencia y cantidad de consumo de alcohol con la edad $\left(r_{s}=.156, p=.039\right)$; lo que indica que a mayor edad mayor frecuencia y cantidad de consumo de alcohol. Se observa correlación positiva significativa entre la cantidad de bebidas alcohólicas consumidas por ocasión y la edad $\left(r_{s}=.276, p<.001\right)$, lo que indica que a mayor edad mayor cantidad de bebidas alcohólicas consumidas por ocasión.

Existen diferencias significativas entre el índice de frecuencia de consumo $(U=2988.5, p=.008)$ por sexo, siendo mayor la frecuencia de consumo en el sexo masculino $(\bar{X}=37.4, M d n=33.3)$ que en el femenino $(\bar{X}=27.3, M d n=16.66)$. Respecto a la edad de inicio de consumo de alcohol $(U=4909.0, p=.001)$ existe diferencia significativa entre el sexo masculino $(\bar{X}=12.8, M d n=13)$ y el femenino $(\bar{X}=$ 13.1 ,

$M d n=13$ ) observándose menor edad de inicio en los hombres. En lo que respecta a los valores no se encontró relación significativa entre el índice de valores terminales e instrumentales con el consumo de alcohol $(p<.05)$.

En la tabla 1 se muestran las relaciones del consumo de alcohol con los índices de espiritualidad. Se mostró correlación positiva y significativa entre las creencias espirituales y el índice de consumo dependiente de alcohol $\left(r_{s}=.181, p=.016\right)$, lo que indica que a mayores creencias espirituales mayor es el consumo dependiente de alcohol, se encontró correlación negativa y significativa entre las creencias espirituales y la cantidad de bebidas alcohólicas consumidas por ocasión $\left(r_{s}=-.117, p\right.$ $=.038$ ), lo que indica que a mayores creencias espirituales menor es la cantidad de 
bebidas alcohólicas consumidas por ocasión, por último, se observó que existe correlación positiva y significativa entre las necesidades espirituales y el índice de consumo dañino $\left(r_{s}=.180, p=.017\right)$ lo que significa que a mayores necesidades espirituales mayor es el índice de consumo dañino.

Tabla 1. Coeficiente de Correlación de Spearman de espiritualidad y consumo de alcohol

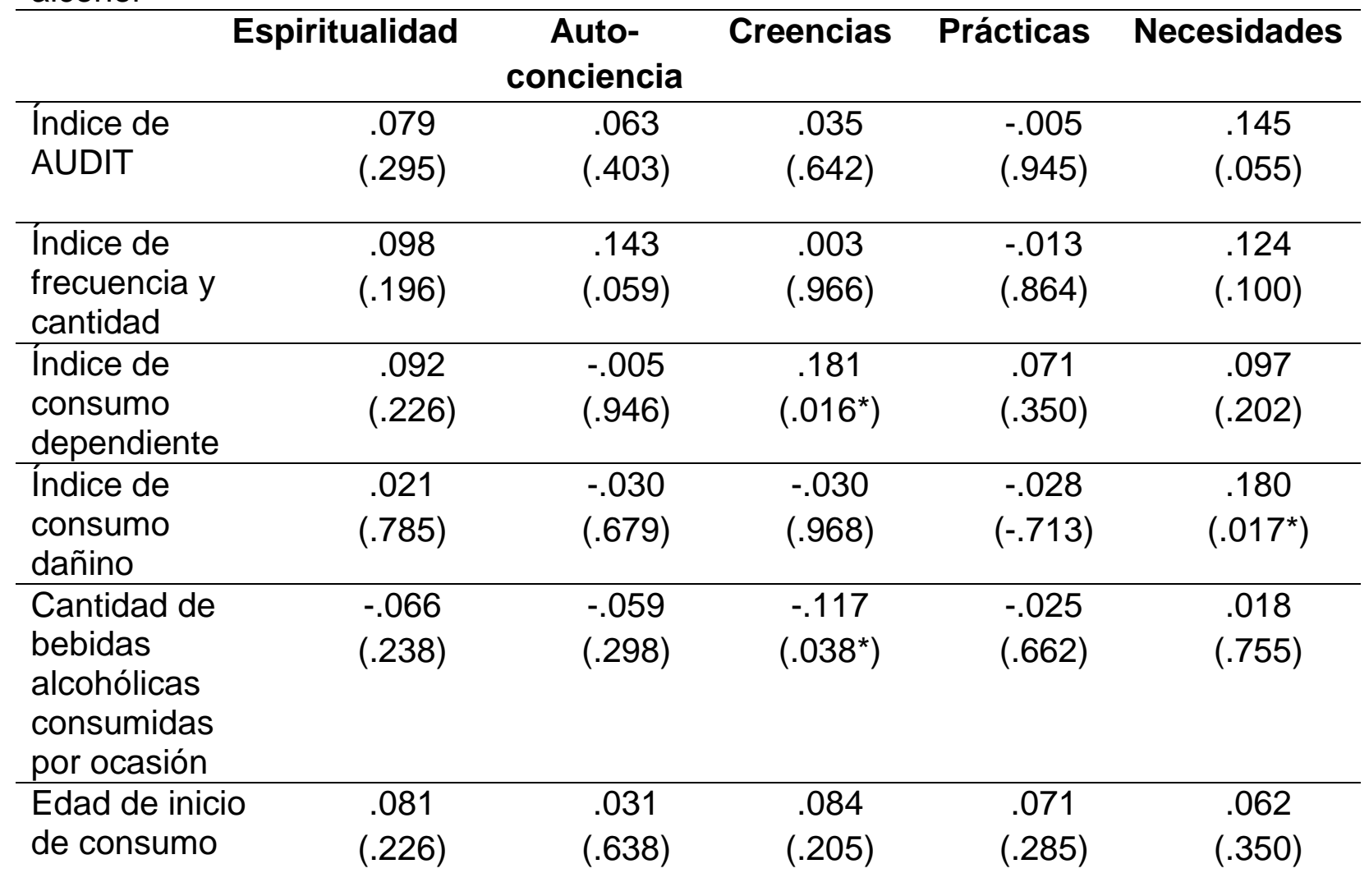

Nota: $p=$ significancia entre paréntesis

\section{DISCUSIÓN}

En relación al propósito del estudio que estableció determinar la relación que existe entre los valores terminales, los valores instrumentales, la espiritualidad y el consumo de alcohol en estudiantes de preparatoria, no se encontró correlación significativa en cuanto al índice de valores terminales e instrumentales y el consumo de alcohol, lo que difiere de lo encontrado por Rodríguez en $2015^{(15)}$ donde el índice de valores terminales se relacionó de manera negativa y significativa con el consumo de alcohol dependiente en estudiantes, lo que puede deberse a que los participantes de este estudio pertenecían a un área suburbana a diferencia de los participantes del presente estudio que pertenecen a un área metropolitana.

En cuanto a la espiritualidad y el consumo de alcohol, se encontró que existe correlación significativa entre las creencias espirituales y el índice de consumo dependiente de alcohol, lo que significa que a mayores creencias espirituales mayor es el índice de consumo de alcohol, esto puede deberse a las restricciones por parte de los grupos religiosos en cuanto al comportamiento de los adolescentes, los adolescentes no desean ajustarse a los valores y normas generales de la sociedad y esto puede considerarse parte del proceso de maduración en esta etapa ${ }^{(16)}$. 
Se encontró también correlación negativa significativa entre las creencias espirituales y la cantidad de bebidas alcohólicas por ocasión, lo que significa que a mayores creencias espirituales menor es la cantidad de bebidas alcohólicas consumidas por ocasión, lo que puede deberse a que quienes tienen mayores creencias espirituales tienden a conductas saludables, si bien consumen no lo hacen de forma riesgosa 0 dañina ${ }^{(17)}$. Así mismo se observó que existe correlación significativa entre las necesidades espirituales y el índice de consumo dañino lo que significa que a mayores necesidades espirituales mayor es el consumo dañino, lo que puede deberse a que la subescala de necesidades espirituales está centrada en el yo y solo hace referencia a la definición de preferencias y búsqueda de intereses personales, y no a las premisas espirituales como el buscar el bien del otro antes que el propio ${ }^{(18)}$.

\section{CONCLUSIONES}

Debido a los resultados previamente presentados concluimos que ciertos componentes de la espiritualidad podrían fungir como factores protectores para el consumo de alcohol, por otro lado, y difiriendo con la literatura hay componentes que se relacionaron con un mayor consumo, por ello se propone continuar utilizando el cuestionario de Parsian y Dunning (2009) para identificar si los resultados se comportan de la misma forma en poblaciones con características diferentes.

Se desconoce la forma que los valores y la espiritualidad modifican las conductas de los adolescentes en cuanto al consumo y no consumo de alcohol por ello se recomienda continuar realizando estudios que indaguen acerca de las variables del presente pero de una forma cualitativa en los adolescentes, ya que estos conceptos resultan ser abstractos y profundos y la interpretación de ellos puede resultan en información que posteriormente permita definirlos de forma adecuada. También se recomienda realizar estudios comparativos en población no escolarizada para identificar si hay diferencias a lo reportado en este estudio.

\section{REFERENCIAS}

1. Organización Mundial de la Salud [OMS]. Global Status report on alcohol and health [Internet]. 2014 [citado 7 mar 2016]; Disponible en: http://apps.who.int/iris/bitstream/10665/112736/1/9789240692763_eng.pdf

2. Instituto Nacional de Salud Pública. Encuesta Nacional de Salud y Nutrición [Internet]. 2012 feb [citado 23 feb 2016]. Disponible en: http://ensanut.insp.mx/informes/ENSANUT2012ResultadosNacionales.pdf

3. Instituto Nacional de Psiquiatría Ramón de la Fuente Muñiz; Instituto Nacional de Salud Pública; Comisión Nacional Contra las Adicciones; Secretaría de Salud. Encuesta Nacional de Consumo de Alcohol, Drogas y Tabaco 2016-2017: Reporte de Alcohol. [Internet] Disponible en: https://www.gob.mx/salud\%7Cconadic/acciones-yprogramas/encuesta-nacional-de-consumo-de-drogas-alcohol-y-tabaco-encodat-20162017-136758

4. Martínez, D, Pallarés, J. (2013). De Riesgos y Placeres. Manual para entender las Drogas. Lleida, España. Primera Edición. Editorial Milenio, 23-36.

5. Rodríguez L A, Alonso M M, Álvarez J, Gómez M V, Armendáriz N A, Hernández E K. Perspectiva espiritual en integrantes de alcohólicos anónimos. 2017;47.

6. Porche P, Fortuna L, Wachholtz A, Torres R. Distal and Proximal Religiosity as Protective Factors for Adolescent and Emerging Adult Alcohol Use. Religions. [Internet] [Citado en 20 marzo] 20172015;6(2): 368-384. Disponible en: http://www.mdpi.com/journal/religions 
7. Kurdai L, Taha A, Dee V. Substance Abuse in Middle Eastern Adolescents Living in Two Different Countries: Spiritual, Cultural, Family and Personal Factors. Journal of Religion and Health. 2014;53: 1060-1074.

8. Páramo M.A. Factores de Riesgo y Factores de Protección en la Adolescencia: Análisis de Contenido a través de Grupos de Discusión. Terapia psicológica. 2011;29(1); 85-95. Disponible en: http://teps.cl/index.php/teps

9. Salas W.C, Olate R, Vaughn M. Religious Coping, Spirituality, and Substance Use and Abuse Among Youth in High-Risk Communities in San Salvador, El Salvador. Substance Use \& Misuse. [Internet] 2014 [citado 15 Marzo 2016]..;48(2): 791-805. Disponible en: http://www.tandfonline.com/loi/isum20

10. Knight J R, Sherritt L, Kim S, Holder D W, Kulig J, Shrier, L.A...Chang G, Alcohol Use and Religiousness/Spirituality Among Adolescents. Southern Medical Journal. 2007;100(4): 349-355.

11. Caravaca J A, Noh S, Hamilton H, Brands B, Gastaldo D, \& Miotto M G. Factores socioculturales y consumo de drogas entre estudiantes universitarios Costarricenses. Texto \& Contexto Enfermagem. 2015; 24, 145-153. Disponible en: http://www.redalyc.org/articulo.oa?id=71439758018

12. McDonald K L, Benish-Weisman M, O'Brien, C T, \& Ungvary S. The Social Values of Aggressive-Prosocial Youth. Journal of Youth Adolescence. [Internet] 2015;44: 2245-2256. Disponible en: https://mijn.bsl.nl/the-social-values-of-aggressive-prosocialyouth $/ 586602$

13. Schwartz SH, \& Blisky W.Towards a universal psychological structure of human values. Journal of Personality and Social Psychology. 1987. 53: 550-562.

14. Parsian N, \& Dunning T. Developing and Validating a Questionnaire to Measure Spirituality: A Psychometric Process. Global Journal of Health Sciencie. 2009;1(1).

15. Rodríguez L A, Alonso B, Alonso M M, Alonso M T, Oliva N N, \& Armendáriz N A. Valores terminales, valores instrumentales, y consumo de alcohol y tabaco en estudiantes de preparatoria. Enfermería Comunitaria. 2015;11(1): 1-7.

16. Fondo de las Naciones Unidas para la Infancia (UNICEF). Estado Mundial de la Infancia 2011. La adolescencia, Una época de oportunidades. 2011. Disponible en: http://www.unicef.org/mexico/spanish/SOWC-2011-MainReport SP 02092011.pdf

17. Burke A, Van J, Eliason M, Howell R, \& Gonzalez. Re-examining religiosity as a protective factor: Comparing alcohol use by self-identified Spiritual, and Secular College Students. Journal of Religion and Health. 2014;53:306-316.

18. Katho, N, \& Sgoutas-Emch, S. Alcohol Use in College: The Relationship Between Religion, Spirituality, and Proscriptive Attitudes Toward Alcohol. Journal of Religion and Health. 2017; 56(2), 437-449.

ISSN 1695-6141

(c) COPYRIGHT Servicio de Publicaciones - Universidad de Murcia 